\title{
C57BL/6 background attenuates mHTT toxicity in the striatum of YAC128 mice
}

\author{
Michaela Kerstin Back ${ }^{1,2}$, Johanna Kurzawa ${ }^{1,2}$, Sonia Ruggieri ${ }^{1}$ and Jakob von Engelhardt $1, *$ \\ 1 Institute of Pathophysiology, Focus Program Translational Neuroscience (FTN), University Medical Cen- \\ ter of the Johannes Gutenberg, University Mainz, 55128 Mainz, Germany; michaela.back@uni-mainz.de \\ (M.K.B.); kurzawa@uni-mainz.de (J.K.); rugsonia@uni-mainz.de (S.R.) \\ 2 These authors contributed equally \\ * Correspondence: engelhardt@uni-mainz.de
}

\begin{abstract}
Mouse models are frequently used to study Huntington's disease (HD). Onset and severity of neuronal and behavioral pathologies vary greatly between HD mouse models, which results from different huntingtin expression levels and different CAG repeat length. HD pathology appears to depend also on strain background of mouse models. Thus, behavioral deficits of HD mice are more severe in the FVB than in the C57BL/6 background. Alterations in medium spiny neuron (MSN) morphology and function has been well documented in young YAC128 mice in the FVB background. We here tested the relevance of strain background for mutant huntingtin (mHTT) toxicity on the cellular level by investigating HD pathologies in YAC128 mice in the C57BL/6 background (YAC128/BL6). Morphology, spine density, synapse function and membrane properties were not or only subtly altered in MSNs of 12-month-old YAC128/BL6 mice. Despite the mild cellular phenotype, YAC128/BL6 mice showed deficits in motor performance. More pronounced alterations in MSN function were found in the HdhQ150 mouse model in the C57BL/6 background (HdhQ150/BL6). Consistent with the differences in HD pathology, the number of inclusion bodies was considerably lower in YAC128/BL6 mice than HdhQ150/BL6 mice. This study highlights the relevance of strain background for mHTT toxicity in HD mouse models.
\end{abstract}

Keywords: Huntington's disease, YAC128, HdhQ150, strain background, C57BL/6, synaptic pathology, extrasynaptic NMDAR

\section{Introduction}

Huntington's disease (HD) is an autosomal dominant neurodegenerative disorder. HD patients show symptoms like motor-dysfunction, starting with involuntary movement and leading to complete loss of intended motion at later disease stages, cognitive deficits and psychiatric symptoms [1]. Causal for many of these symptoms is neurodegeneration of the striatum with apoptosis of GABAergic medium spiny neurons (MSNs) (reviewed in [2]). Later during disease progression, also other brain areas are affected such as the cerebral cortex [3].

$\mathrm{HD}$ is a trinucleotide repeat disorder, which is caused by prolonged CAG repeats $(>35)$ in exon one of the huntingtin gene (HTT). Disease severeness correlates with the length of the trinucleotide expansion [4]. The physiological function of the huntingtin protein (HTT) includes transcriptional regulation, transport of vesicles and synaptic activity [5, 6]. Mutant HTT (mHTT), however, disrupts these cellular processes and neuronal functions leading to neuronal death (for review, see [7]).

HD mouse models are important tools for studying the underlying pathomechanisms and for the search of therapeutic strategies. The functional and structural analysis of brains of these mouse models as well as brains of HD patients suggests that neuronal dysfunction with alterations in synaptic function and number occurs before cell death $[8$, 9]. There exist several HD mouse models including transgenic and knock-in HD mouse 
models, which express human or mouse mHTT [10]. An extensively studied HD murine model is the YAC128 mouse [11]. This mouse model codes for the entire human HTT on a yeast artificial chromosome (YAC) under control of the human promoter [11]. YAC128 mice display several important pathologies that are also observed in HD patients with synapse dysfunction and spine loss in MSNs of 3-month-old mice [12], neuronal loss in the striatum at 12 months of age [11], and motor dysfunction in 4-month-old mice [13-15]. The striatum in YAC128 mice is the earliest but not exclusively affected brain region [16]. Due to their comparably mild and protracted phenotype, YAC128 mice are described as an ideal mouse model for studying adult-onset HD [17]. In contrast, the rapid disease progression in another mouse model, namely R6, makes it a model of juvenile HD [17]. Variability in onset and severity of pathology in HD mouse models can be explained by the different types of HTT with varying length of the CAG repeats and the different HTT expression levels (reviewed in [18]). However, strain background may contribute to these differences similar as in other neurodegenerative disease mouse models. Thus, the A $\beta$ level in the Alzheimer's disease mouse model 5xFAD was shown to depend on the genetic background [19]. Impacts of the background strain have been described also in pain mouse models [20]. This relevance of the background strain is mainly explained by the strain-specific activity of so-called modifier-genes that influence the impact of specific gene mutations (reviewed in [21]).

YAC128 mice were generated in the FVB/N background [11] and most studies use YAC128 mice with this background [22-29]. However, the FVB/N strain is not always the optimal background. For example, FVB/N mice have retinal degeneration, which complicates many behavioral assays. Thus, YAC128 mice were used in the C57BL/6 or the 129 background by sub-breeding YAC128 mice with these strains or by sub-breeding with other transgenic or knockout mouse lines [13, 30-33]. Differences in strain background may well contribute to variability of disease onset and severity in studies using YAC128 mice. Indeed, severity of motor dysfunction and magnitude of the reduction in striatal volume depend on the background with the strongest pathologies in YAC128 mice with the FVB/N background and comparably milder phenotype in mice with C57BL/6 background. Mice with 129 background showed intermediate phenotype severity [13]. Similar, the dependency of onset and severity of behavioral deficits on the strain background was demonstrated in another study that investigated different HD mouse models including R6/2, HdhQ111, BACHD and YAC128 mice [34]. Importantly, behavior itself such as motor performance on the Rotarod, activity and rearing in the Open Field test, anxiety, and startle response is very variable in wild type mice with different background ( $\mathrm{FVB} / \mathrm{N}$ and C57BL/6) [34]. Thus, the interpretation that the variability of behavioral deficits in YAC128 mice results from strain-specific differences in mHTT toxicity is somewhat confounded by strain-specific differences in behavior.

We therefore wondered whether the comparably mild behavioral deficits in YAC128 mice in C57BL/6 background is reflected by resistance of MSNs to mHTT toxicity. YAC128 mice have frequently been used to investigate early pathologies of HD such as spine loss, synaptic dysfunction, and alterations in passive electrophysiological properties. These pathologies have been well documented in 3-12-month-old YAC128 mice with FVB/N background [12, 35].

In this study, we investigated spine density and dendritic arborization, synaptic function, and passive electrophysiological properties of MSN from YAC128 mice in the C57BL/6N background. We observed comparably mild alterations in YAC128/BL6 mice even at an age of one year. As described previously, motor behavior was altered in YAC128/BL6 mice. In order to study, whether the C57BL/6 strain background per se averts $\mathrm{mHTT}$ toxicity, we used the HdhQ150 mouse model, which has been generated in a mixed 129/Ola and C57BL/6 background [36] and is frequently used in a 75-90\% C57BL/6 background [10]. Synapse dysfunction was observed in 6- month-old HhdQ150/BL6 mice. Consistent with the difference in mHTT toxicity, we observed a considerably higher number of inclusion bodies in the striatum of HhdQ150/BL6 than in the striatum of YAC128/BL6. 
In summary, our results suggest that strain background in combination with the propensity to form inclusion bodies influences severity and onset of disease pathology in the striatum of HD mouse models.
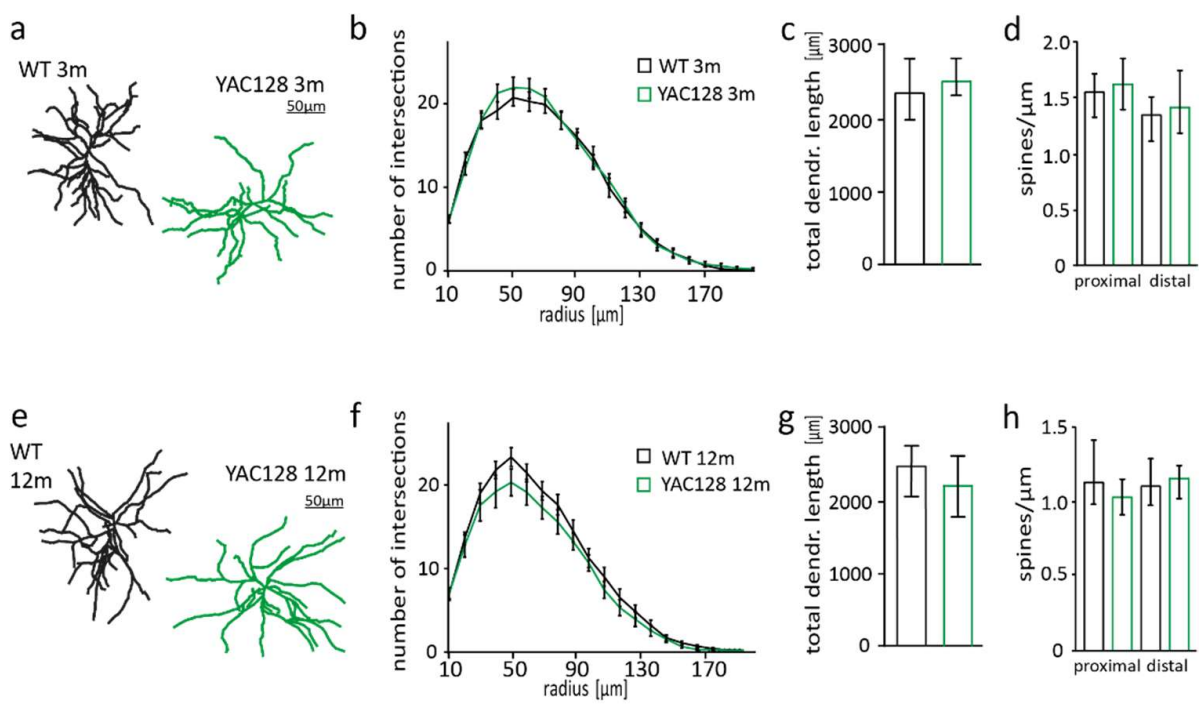

Figure 1: Neuron morphology is unaltered in 3- and 12-month-old YAC128/BL6 mice. (a) Examples of reconstructed medium spiny neurons (MSNs) from 3-month-old WT and YAC128/BL6 mice. (b) Sholl analysis with number of dendrite intersections and (c) bar graph of total dendritic length of MSNs from WT (n=23) and YAC128/BL6 (n=23) mice. (d) Bar graph of spine number per micrometer of proximal and distal dendrites of MSNs from WT (proximal $n=16$, distal $n=17$ ) and YAC128/BL6 (proximal n=27, distal n=29) mice. (e) Examples of reconstructed of MSNs from 12month-old WT and YAC128/BL6 mice. (f) Sholl analysis with number of dendrite intersections and (g) bar graph of total dendritic length of MSNs from WT (n=18) and YAC128/BL6 (n=15) mice. (h) Bar graph of spine number per micrometer of proximal and distal dendrites of MSNs from WT (proximal $n=29$, distal $n=27$ ) and YAC128/BL6 (proximal $n=27$, distal $n=29$ ) mice. Bar graphs are depicted as median \pm IQR.

\section{Results}

\subsection{Normal neuron morphology and spine number in 12-month-old YAC128/BL6 mice}

Morphological changes in MSNs precede neuron loss and are an early indicator of HD pathology [37]. Spine density is mildly reduced in 3-4 month-old YAC128 and strongly reduced by more than $30 \%$ in YAC128/FVB mice at an age of 12 months [12, 28, $38,39]$. To investigate the relevance of the strain background on mHTT toxicity, we quantified spine number in YAC128 mice in the C57BL/6 background (YAC128/BL6). The number of spines in proximal and distal dendrites of MSNs were not different between 3month-old YAC128/BL6 mice and control littermates (proximal: $t$-test; $p=0.6939$; distal: $t$ test; $\mathrm{p}=0.1947$ ) (Figure 1d). Spine number was also not reduced in MSNs of 12-month-old YAC128/BL6 mice (; t-test; $p=0.0697$ ) (Figure 1h). Sholl analysis of biocytin-filled and reconstructed MSNs revealed no difference in dendritic arborization as well as total dendritic length between 3-month or 12-month-old WT and YAC128/BL6 mice (t-test; 3 months: $\mathrm{p}=0.311 ; 12$ months: $\mathrm{p}=0.1775$ ) (Figure 1a-c+e-g). Thus, we observed no changes in MSN morphology in both 3-month and 12-month-old YAC128/BL6 mice in contrast to the alterations in MSN morphology with reduced spine density in 3- and 12-month-old YAC128/FVB mice [12, 38, 39].

\subsection{Subtle changes in synapse function in 12-month-old YAC128/BL6 mice.}


Synaptic dysfunction of MSNs occurs early in HD patients and in HD mouse models $[8,40,41]$. Thus, alterations in synaptic function are present in YAC128 mice with FVB background already at an age of 1.5 months [42-44]. At the age of 12 months and older, synaptic function of MSNs from YAC128/FVB mice is severely disturbed $[42,43,45]$. To investigate whether the strain background affects the occurrence of synaptic dysfunction in YAC128 mice, we recorded $\alpha$-amino-3-hydroxy-5-methyl-4-isoxazolepropionic acid receptor (AMPAR)-mediated miniature excitatory postsynaptic currents (mEPSCs) in striatal MSNs of YAC128/BL6 and WT mice. Neither mEPSC frequency nor amplitude were different between genotypes in 3-month-old mice (Suppl. Figure 1b). mEPSC frequency was also not affected in 12-month-old YAC128/BL6 mice (there was a trend to reduced frequency in YAC128/BL6 mice; Mann-Whitney test, $\mathrm{p}=0.435)$ (Figure 2a+b). There was a small but significant increase in mEPSC peak amplitude in MSNs from YAC128/BL6 mice ( $\mathrm{t}$-test, $\mathrm{p}=0.021$ ) (Figure $2 \mathrm{a}+\mathrm{b}$ ). Considering the trend to reduced mEPSC frequency in YAC128/BL6 mice, we wondered whether there is a significant reduction in older YAC128/BL6 mice. However, neither mEPSC frequency nor amplitude were altered in 18month-old YAC128/BL6 mice when compared to WT littermates (Suppl. Figure 1d).

Presynaptic function with altered vesicle release probability has been observed in YAC128/FVB mice. Thus, the paired-pulse ratio (PPR), which changes with alterations in presynaptic vesicle release probability, is decreased in 1.5-month-old mice and increased in 12-month-old YAC128/FVB mice [42]. To investigate presynaptic function, we investigated PPR of evoked EPSCs with an inter-stimulus-interval of 50 ms. PPR was slightly reduced in MSNs of 12-month-old YAC128/BL6 mice compared to their littermate controls (t-test; $\mathrm{p}=0.0426$ ) (Figure 2c). The decrease in PPR suggests that vesicle release probability is reduced in YAC128/BL6 mice and may explain the trend to reduced mEPSC frequency. Interestingly, the reduced PPR in 12-month-old YAC128/BL6 mice resembles the PPR change in 1.5-month-old YAC128/FVB mice and is opposite to the increase in PPR in 12month-old YAC128/FVB mice [42].

$\mathrm{N}$-methyl-D-aspartate receptor (NMDAR) expression increases and function changes in HD mouse models [46]. Expression of NMDARs increases in the striatum of YAC128 mice already at an age of one month prior to occurrence of neuropathology and behavioral deficits $[47,48]$. These alterations in NMDAR expression and function are considered to play an important role in HD pathophysiology $[49,50]$. Synaptic NMDAR currents are increased in 1-month and 12-month-old YAC128 mice with FVB background [48]. To assess synaptic NMDAR function, we recorded evoked NMDAR-mediated currents in MSNs. The amplitude of NMDAR-mediated currents was normalized to the amplitude of AMPAR-mediated currents. The NMDA/AMPA-ratio was unaltered in YAC128/BL6 mice (Mann-Whitney test; $\mathrm{p}=0.4102$ ) (Figure 2d) suggesting that the number of synaptic NMDARs relative to the number of synaptic AMPARs is not altered in MSNs of 12-monthold YAC128/BL6 mice. Considering that AMPAR-mediated EPSC amplitudes were only slightly increased (Figure 2a), this indicates that there may be a small increase in the number of synaptic NMDARs. NMDARs of striatal MSNs are known to contain mainly GluN2A and GluN2B subunits [51,52]. The composition of NMDARs determines current kinetics. GluN2B-containing NMDARs deactivate considerably slower than GluN2A-containing NMDARs [53]. Similar decay kinetics of synaptic NMDAR-mediated currents in MSNs of YAC128/BL6 and WT mice (Figure 2e, t-test; $p=0.7485$ ) suggest that NMDAR subunit composition is not different between genotypes. 
a

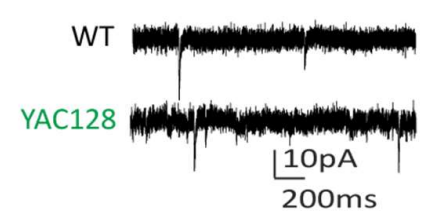

C

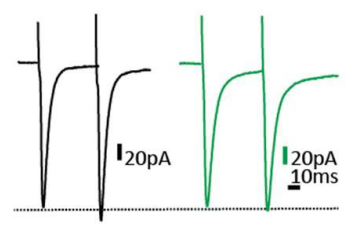

d

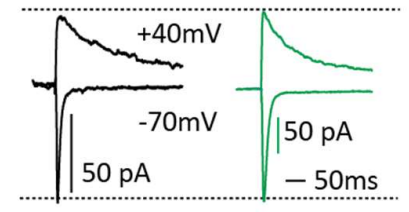

$\mathrm{b}$
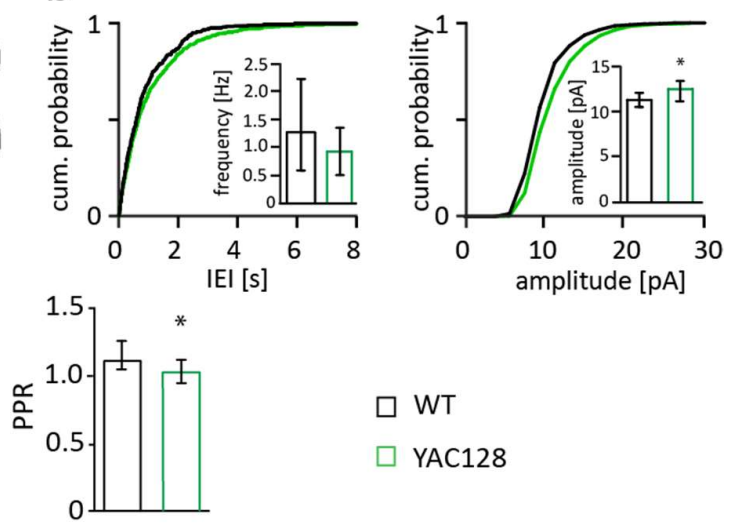

WT

YAC128

e

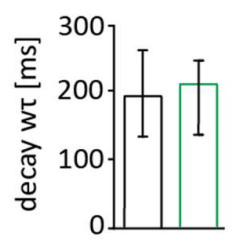

f
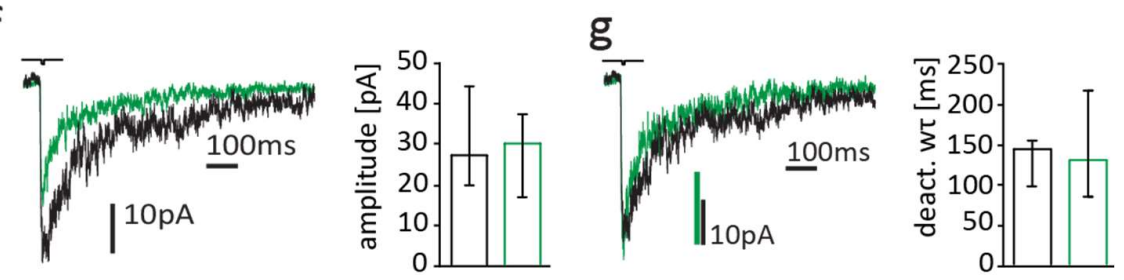

Figure 2: Synapse function is subtly changed in 12-month-old YAC128/BL6 mice. (a) Sample traces of miniature excitatory postsynaptic currents (mEPSC) recordings from MSNs of WT and YAC128/BL6 mice. (b) Cumulative distribution plot of AMPAR-mediated miniature excitatory postsynaptic currents (mEPSC) inter-event-intervals (IEIs) and amplitude. The inserts show bar graphs of the median mEPSC frequency and amplitude of MSNs from WT (black, n=24) and YAC128/BL6 (green, $n=26$ ) mice. (c) Sample traces of paired-pulse (PP) recordings from MSNs of WT (black) and YAC128/BL6 mice (green). Currents were evoked with an inter-pulse-interval of $50 \mathrm{~ms}$. Bar graph of PP ratio (PPR) in MSNs of WT ( $n=9)$ and YAC128/BL6 ( $n=13)$ mice. (d) Sample traces of NMDAR- and AMPAR-mediated currents recorded at a holding potential of $+40 \mathrm{mV}$ and $-70 \mathrm{mV}$, respectively. Bar graph of NMDA/AMPA ratio of MSNs from WT $(\mathrm{n}=19)$ and YAC128/BL6 $(\mathrm{n}=15)$ mice. (e) Bar graph of the weighted time constant $(\mathrm{w} \tau)$ of synaptic NMDAR-mediated current decays of MSNs from WT $(n=18)$ and YAC128/BL6 (n=15) mice. (f) Sample traces of extrasynaptic NMDAR-mediated currents recorded from nucleated patches of MSNs and bar graph of median peak current amplitudes of MSNs from WT (n=13) and YAC128/BL6 (n=18) mice. (g) Peaknormalized sample traces of extrasynaptic NMDAR-mediated currents recorded from nucleated patches of MSNs and bar graph of the weighted time constant $(\mathrm{w} \tau)$ of current deactivation in nucleated patches of MSNs of WT $(n=10)$ and YAC128/BL6 $(n=14)$ mice. Bar graphs are depicted as median \pm IQR.

The expression of extrasynaptic GluN2B-containing NMDARs increases in MSNs of 1-month-old YAC128 with FVB background [47, 48]. This increase is thought to be relevant for HD pathophysiology [50] as the activation of extrasynaptic GluN2B-containing NMDARs induces cell-death pathways [54,55]. We activated extrasynaptic NMDARs by ultra-fast application of glutamate onto nucleated patches of MSNs. Amplitude and deactivation kinetics of extrasynaptic NMDAR-mediated currents were not affected in in MSNs of 12-month-old YAC128/BL6 mice (t-test; $\mathrm{p}=0.349$ and $\mathrm{p}=0.5273$, respectively) (Figure $2 \mathrm{f}, \mathrm{g}$ ) indicating that number and composition of extrasynaptic NMDARs are not different between genotypes. 
Thus, the electrophysiological analyses revealed only minor alterations in synaptic function and no change in NMDAR-mediated currents in MSNs of 12-month-old YAC128/BL6 mice.

\subsection{Subtle changes in active membrane properties of MSNs in 12-month-old YAC128/BL6 mice.}

Electrophysiological membrane properties of MSNs are affected in 7 to 12-month-old YAC128 mice in the FVB background [35,47]. We studied basic membrane properties in MSNs from 12-month-old YAC128/BL6 mice and littermate controls. There was no genotype difference in input resistance (Mann-Whitney test; $\mathrm{p}=0.748$ ) and membrane potential (t-test; $\mathrm{p}=0.4122$ ) (Figure 3b). Also, action potential threshold (t-test; $\mathrm{p}=0.2182$ ) and amplitude ( $\mathrm{t}$-test; $\mathrm{p}=0.4666$ ) were not affected in MSNs from 12-month-old YAC128/BL6 mice (Figure 3b). Action potential duration was slightly decreased in MSNs from YAC128/BL6 mice (Mann-Whitney test; $\mathrm{p}=0.0297$ ) (Figure 3b).

Firing properties of YAC128/BL6 MSNs were analyzed by maximal current injections. Maximal firing frequency ( $t$-test; $\mathrm{p}=0.1392$ ), early spike adaptation ( $\mathrm{t}$-test; $\mathrm{p}=0.1415$ ) and late spike adaptation ( $\mathrm{t}$-test; $\mathrm{p}=0.539$ ) were not altered in MSNs from 12-month-old YAC128/BL6 mice (Figure 3d). This indicates that passive and active membrane properties of MSNs are only mildly affected in 12-month-old YAC128/BL6 mice.

\subsection{Early synaptic deficits in MSNs of HdhQ150/BL6 mice}

The analyses with YAC128 mice showed that alterations of MSN morphology and function are very mild in the C56BL/6 background. We wondered whether that holds true also for mice with higher mHTT expression. The HdhQ150 mouse model is a well-studied HD model that is bred in the C57BL/6 background but nevertheless shows early behavioral deficits [56]. Morphological analysis revealed that overall dendritic length of MSNs was not altered in 6-month-old HdhQ150/BL6 mice compared to WT littermates (Figure 4c). However, Sholl analysis revealed subtle changes in neuronal arborization with an increase in the number of intersections per radius at distal dendritic compartments of MSNs in HdhQ150/BL6 mice (2-way-ANOVA with Sidak's multiple comparison test) (Figure $4 b)$. Spine number was not affected in proximal (t-test; $p=0.5096$ ) and distal (t-test; $\mathrm{p}=0.6851$ ) dendrite compartments of MSNs in HdhQ150/BL6 mice (Figure 4d).

Electrophysiological analysis revealed a reduced mEPSC frequency ( $t$-test; $p=0.0331$ ) and a corresponding right-shift of the cumulative distribution curve for mEPSC interevent intervals in MSNs from HdhQ150/BL6 mice (Figure 4e+f). mEPSC amplitudes were not different between genotypes (t-test; $\mathrm{p}=0.7829$ ) (Figure $4 \mathrm{f}$ ). The reduction in mEPSC frequency suggests that functional synapse number is reduced in HdhQ150/BL6 mice which is consistent with previous observations in R6/2 and Q175 mice $[57,58]$. 
a

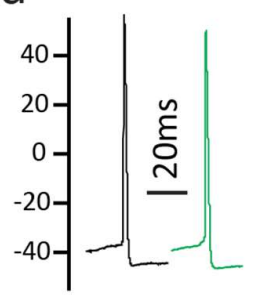

$\square$ WT $\square$ YAC128

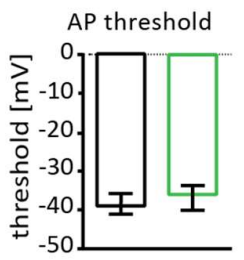

b
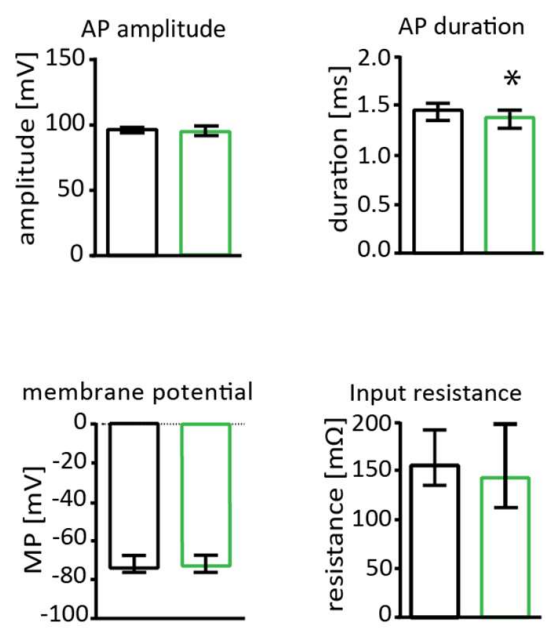

Input resistance

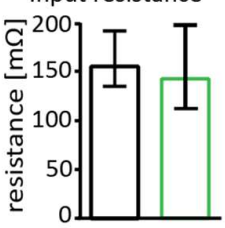

$\mathrm{C}$

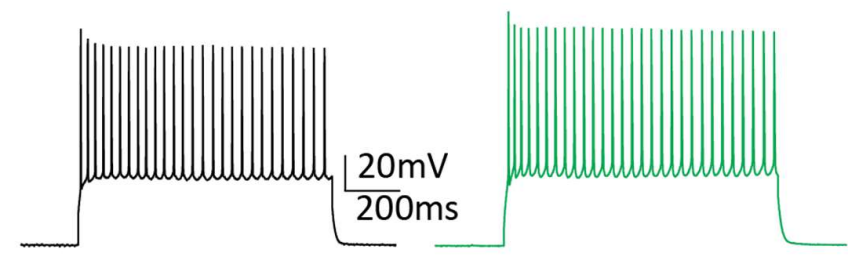

d
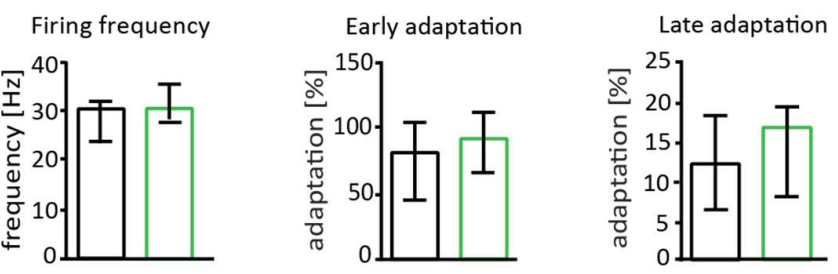

Figure 3: Membrane properties are by and large unaltered in 12-month-old YAC128/BL6 mice. (a) Sample traces of action potentials (APs) recorded from MSNs of WT (black) and YAC128/BL6 (green) mice. (b) Bar graphs of AP threshold, amplitude, threshold and input resistance (WT n=24; YAC128/BL6 n=23). (c) Sample traces of AP firing induced by current injection. (d) Bar graphs of firing frequency, early and late adaptation of MSNs from WT $(n=24)$ and YAC128 $(n=23)$ mice. $\mathrm{MP}=$ membrane potential. Bar graphs are depicted as median $\pm \mathrm{IQR}$.

Importantly, the NMDAR/AMPAR-ratio was increased in MSNs of 6-month-old HdhQ150/BL6 mice compared to that in WT mice (t-test; $\mathrm{p}=0.0187$ ) (Figure 4g). Considering that the amplitude of AMPAR-mediated mEPSCs did not change, these data suggest that the expression of synaptic NMDARs is increased in MSNs of HdhQ150/BL6 mice.

The decay of NMDAR-mediated currents was not affected (t-test; $p=0.2411$ ) (Figure 4h) indicating that the composition of synaptic NMDARs is not strongly altered in MSNs of HdhQ150/BL6 mice. There was, however, a trend to a reduced decay time constant, which may indicate an increased contribution of GluN2A-containing NMDARs. Extrasynaptic NMDAR-mediated currents were not affected in MSNs of HdhQ150/BL6 mice (Figure 4i). Thus, peak amplitude (Mann-Whitney test; $\mathrm{p}=0.9205$ ) and deactivation time constant of NMDAR-mediated currents (Mann-Whitney test; $\mathrm{p}=0.211$ ) were similar between genotypes (Figure $4 \mathrm{i}+\mathrm{j}$ ). 
In summary, we found synaptic and morphological changes in MSNs of 6-month-old HdhQ150 mice. Thus, MSNs of HdhQ150/BL6 mice display more severe and earlier alterations in both MSN morphology and synapse function than MSNs of YAC128/BL6 mice. Moreover, this suggests that the C57BL/6 background provides only partial protection against mHTT toxicity (or protection only in the YAC128 mouse model).

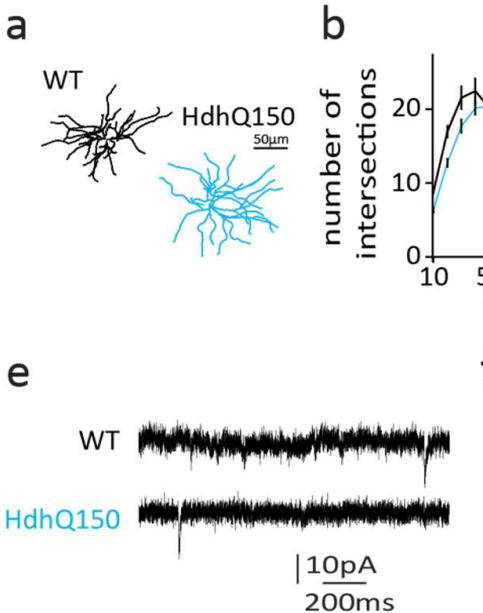

g

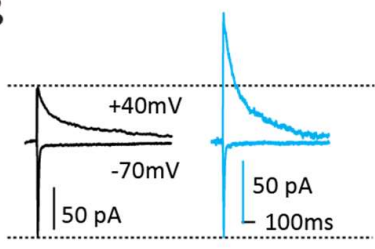

f

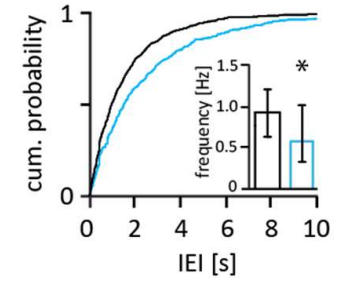

C

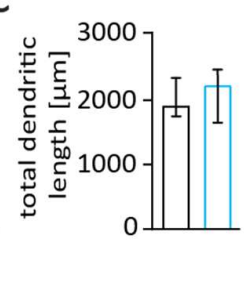

d

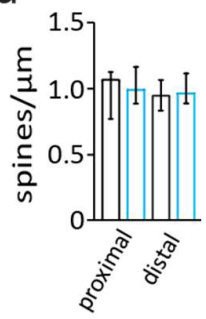

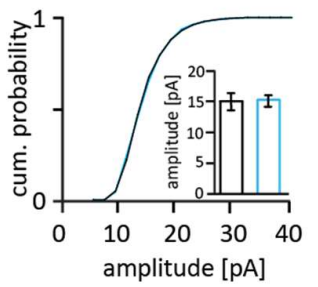

$\mathrm{h}$

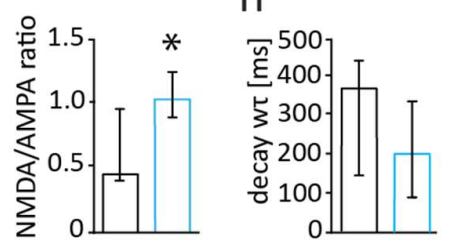

WT

HhdQ150
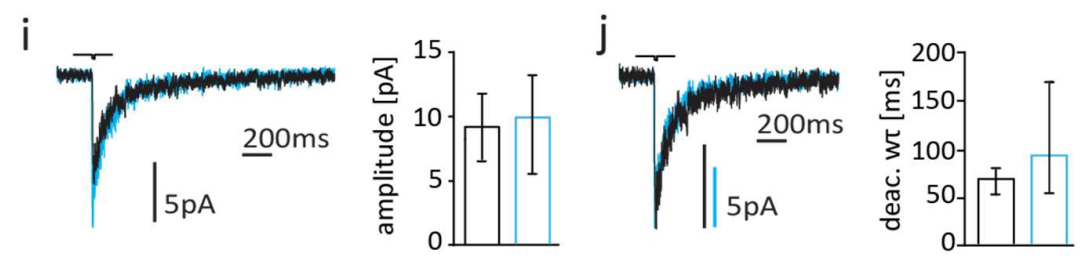

Figure 4: Synaptic function is impaired in 6-month-old HdhQ150/BL6 mice. (a) Example reconstructions of MSNS. (b) Sholl analysis with number of dendrite intersections per radius and (c) bar graph of total dendritic length of MSNs from WT $(n=8)$ and HdhQ150/BL6 ( $n=17)$ mice. (d) Bar graph of spine number per $\mu$ m of MSNs from WT $(n=10)$ and HdhQ150/BL6 ( $n=18)$ mice. (e) Sample traces of mEPSC recordings from MSNs from 6-month-old WT and HdhQ150/BL6 mice. (f) Cumulative distribution plot of mEPSC IEIs and amplitude as well as bar graphs of the median mEPSC frequency and amplitude of MSNs from 6-month-old WT (black, n=31) and HdhQ150/BL6 (blue, $\mathrm{n}=32$ ) mice. (g) Sample traces of NMDAR- and AMPAR-mediated currents recorded at a holding potential of $+40 \mathrm{mV}$ and $-70 \mathrm{mV}$, respectively and bar graph of the NMDA/AMPA-ratio of MSNs from WT $(n=10)$ and HdhQ150/BL6 ( $=15)$ mice. (h) Bar graph of the time constant $(w \tau)$ of NDMAR-mediated current decay in MSNs of WT $(n=9)$ and HdhQ150/BL6 ( $n=15)$ mice (i) Sample traces of extrasynaptic NMDAR-mediated currents recorded in nucleated patches and bar graph of peak current amplitudes of MSNs from WT $(n=9)$ and HdhQ150/BL6 (n=10) mice. (j) Peakscaled sample traces of extrasynaptic NMDAR-mediated currents recorded from nucleated patches and bar graph of the deactivation time constant $(\mathrm{w} \tau)$ of currents from MSNs of WT ( $\mathrm{n}=9$ ) and HdhQ150/BL6 $(\mathrm{n}=10)$ mice. Bar graphs are depicted as median \pm IQR. 
Considering the mild anatomical and functional phenotype in 12-month-old YAC128/BL6 mice, we wondered whether human mHTT is expressed and aggregates in inclusion bodies, which are a hallmark of HD [59]. Interestingly, it was previously shown that the expression level of mHTT is similar in YAC128 mice with different background strains (FVB, C57BL/6 and 129) [13], suggesting that the strain-specific variability in mHTT toxicity does not result from differences in mHTT expression. However, the same study showed that the occurrence of nuclear localization of mHTT, an early hallmark of $\mathrm{HD}$, is different in the three backgrounds strains studied [13]. Thus, nuclear accumulation of mHTT was already found in 1-month-old YAC128/FVB mice, but only from 4 months onwards in YAC128/BL6 mice [13].
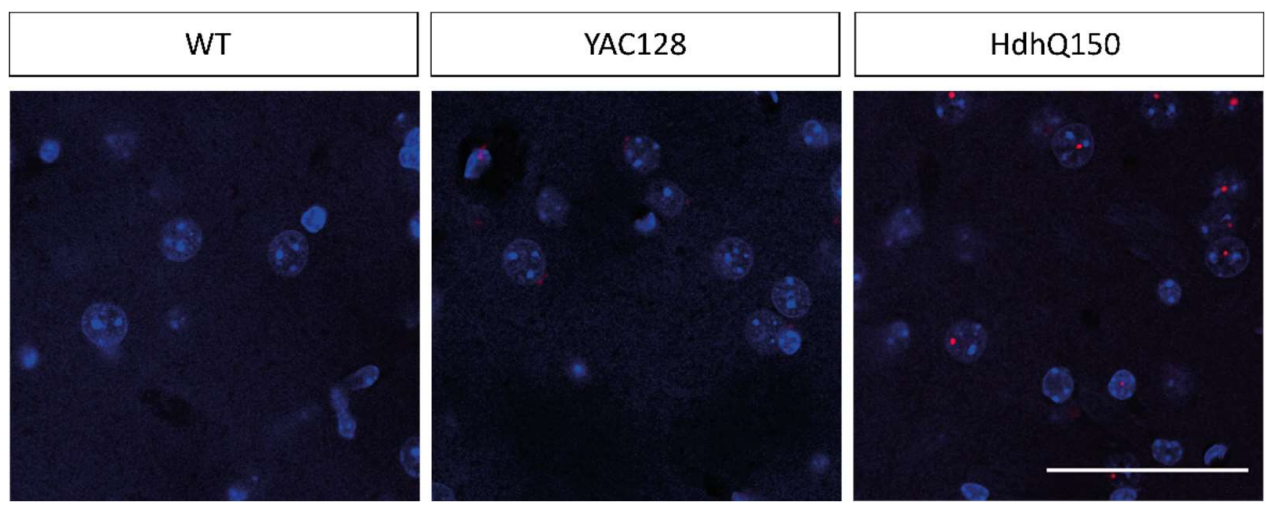

Figure 5: The number of inclusion bodies is lower in YAC128/BL6 than in HdhQ150Q/BL6 mice. Confocal microscopy images of brain slices of the striatum of WT (12 month), YAC128/BL6 (12 month) and HdhQ150 (10 month) mice. Inclusion bodies (red) were visualized with an MW8 antibody and cell nuclei with DAPI staining (blue). Merged images show the inclusion bodies with an intranuclear localization in HdhQ150 animals. Scale bar is $20 \mu \mathrm{m}$ for all confocal images.

We detected few inclusion bodies in the striatum of 12-month-old YAC128/BL6 mice as investigated by immunohistochemistry using an antibody specific for human mHTT (Figure 5). No inclusion bodies were observed in C57BL/6 control mice (WT) (Figure 5). Importantly, the number of inclusion bodies was considerably higher in the brain of HdhQ150/BL6 mice than in the brain of YAC128/BL6 mice (Figure 5).

\subsection{Impaired behavior in YAC128/BL6 mice.}

Considering the low number of inclusion bodies and the subtle alterations in neuron morphology, synaptic function and membrane properties, we wondered whether behavior is affected in YAC128/BL6 mice.

General activity and exploratory behavior were assessed using the Open Field test. No difference was observed in motor activity between 9-month-old YAC128/BL6 and control mice as quantified by the total distance travelled ( $t$-test; $\mathrm{p}=0.7556$ ) (Figure 6a). Mice of both genotypes did also not differ in the distance travelled ( $t$-test; $\mathrm{p}=0.7131$ ), time spent (Mann-Whitney test; $\mathrm{p}=0.0524$ ) and number of visits (t-test; $\mathrm{p}=0.1162$ ) in the periphery of the Open Field (Figure 6a). However, YAC128/BL6 travelled significantly less distance in the center of the Open Field than control mice (t-test; $p=0.004$; Figure 6a). Consequently, the ratio of travelled distance periphery/center was also significantly different between YAC128/BL6 and control mice (t-test; $p=0.0075$; Figure 6a). Despite a lower number of visits of YAC128/BL6 mice in the center ( $t$-test; $\mathrm{p}=0.0061)$, the ratio of visits in periphery/center was not significantly different (t-test; $\mathrm{p}=0.0551$ ) (Figure 6a). Finally, although YAC128/BL6 mice spent a comparable time in the center of the Open Field (Mann-Whitney test; $\mathrm{p}=0.0753$ ), the ratio of time spent in periphery/center was significantly increased 
(t-test; $\mathrm{p}=0.034$; Figure 6a). Findings of the Open Field test suggest that overall motor activity is unaltered but anxiety levels increased in YAC128/BL6 mice.

Motor performance in 9-month-old YAC128/BL6 mice was additionally studied using the Rotarod test. Since body weight can influence the fall latency in Rotarod experiments, we measured the weight of the animals. Male YAC128/BL6 mice had a similar weight compared to male WT mice (Figure 6c), and stayed significantly less time on the Rotarod (t-test; $\mathrm{p}=0.0364$; Figure 6b) compared to male control mice. Although female YAC128/BL6 mice were significantly heavier compared to female WT ( $t$-test; $\mathrm{p}=0.01$ ) (Figure $6 c$ ), fall latency of female mice was not different between genotypes ( $t$-test; $p=0.2873$ ) (Figure 6b).

a Open Field test
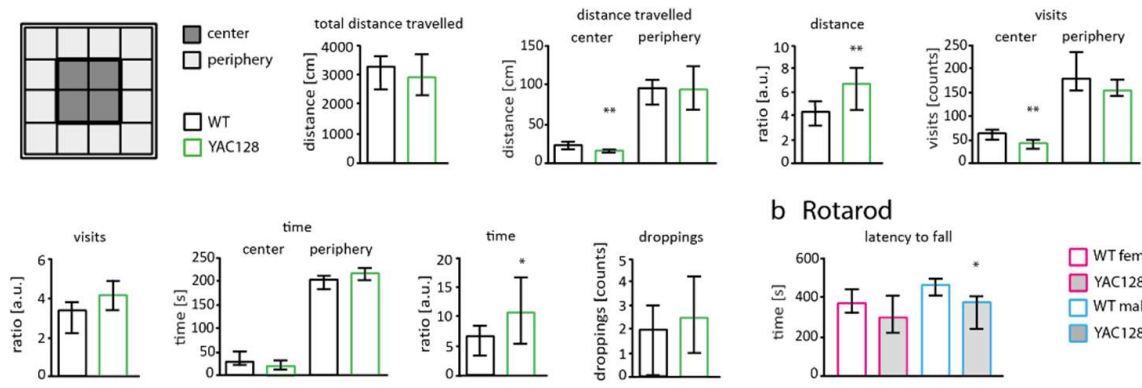

b Rotarod
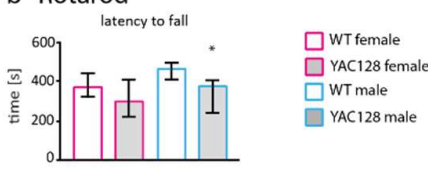
$\square$ WT male

YAC128 male

c

d

e

Body weight

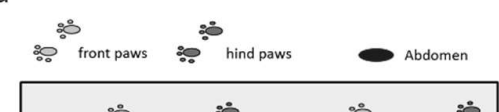
Gait analysis
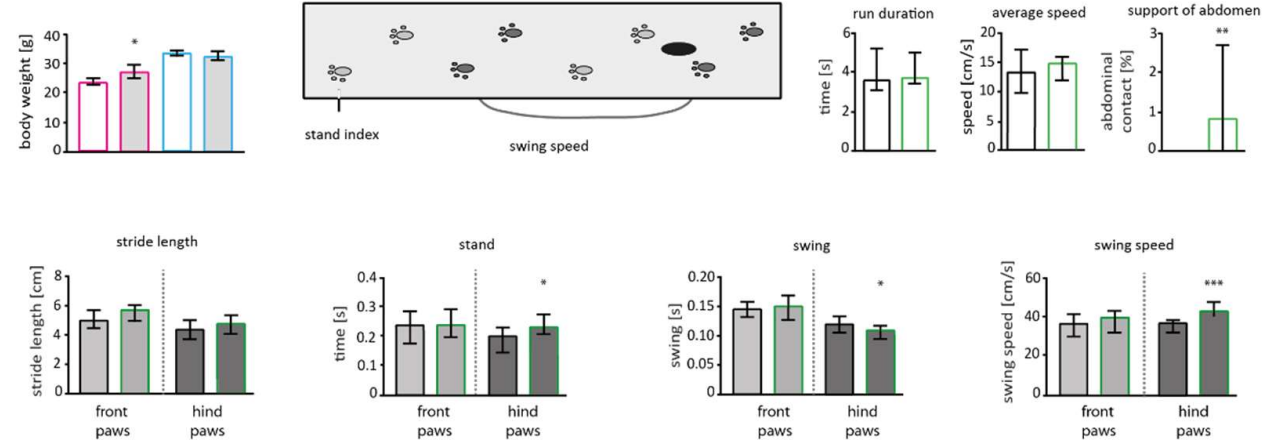

Figure 6: Behavior is impaired in 9-month-old YAC128/BL6 mice. (a - left) Scheme of the Open Field divisions with center and periphery. (a - right) Bar graphs of Open Field behavioral parameters of WT $(n=10)$ and YAC128/BL6 $(n=10)$ mice. (b) Bar graph of the latency to fall off the Rotarod for male and female WT ( $\mathrm{n}$ females = 7; males =6) and YAC128/BL6 ( $\mathrm{n}$ females = 4, males = 5) mice. (c) Bar graph of the median body weight of female and male of WT and YAC128/BL6 mice. (d) Scheme of the gait analysis (Catwalk) setup. (e) Bar graphs of gait analysis parameters from WT $(\mathrm{n}=11)$ and YAC128/BL6 $(\mathrm{n}=12)$ mice. Bar graphs are depicted as median $\pm \mathrm{IQR}$.

Finally, we employed the Catwalk to detect more subtle alterations in motor performance. Average speed and run duration were similar in WT and YAC128/BL6 mice (Figure 6e). YAC128/BL6 mice also showed no deficits in stride length (Figure 6e). We observed an increased swing speed in hind paws of YAC128/BL6 mice (t-test; $\mathrm{p}<0.0001$ ). Consistently, the swing phase time, which describes the time that the paw is in the air, was decreased in hind paws from YAC128/BL6 mice (swing; Mann-Whitney test: $\mathrm{p}=0.0311$ ) (Figure 6e). Stand (time of paw contact with the glass plate) was increased in hind paws of YAC128/BL6 mice (quantified for front and hind paws separately; MannWhitney test: $\mathrm{p}=0.0348$ ) (Figure 6e). We also observed an increased abdominal contact with the glass plate in YAC128/BL6 mice compared to WT mice (Mann-Whitney test; 
$\mathrm{p}=0.0024)$. The increased stand and swing speed of the hind paws together with the abdominal support indicate the need of YAC128/BL6 mice for more support during body movement (Figure 6e).

In summary, behavior analysis of YAC128 mice in the C57BL/6 background revealed motor impairments, consistent with a dysfunction in striatal signaling.

\section{Discussion}

Our data suggest that the strain background affects the phenotype of YAC128 mice. Analysis of MSNs revealed that YAC128 mice in the C57BL/6 background are only mildly affected. This is especially evident for early HD-related perturbations at the synaptic level and less evident for the behavioral phenotype.

HD pathology in humans and mouse models starts with neuronal dysfunction before the onset of cognitive and motor symptoms [9, 50, 60-67]. The underlying mechanisms leading to neuronal and synaptic dysfunction are, however, not yet fully elucidated. Altered NMDAR function and expression in striatal MSNs is thought to be involved [46, 49, 68-73]. It was previously shown, that NMDAR-mediated currents increase in MSNs of HD mouse models, which could lead to excitotoxicity (reviewed in [49, 74]). Specifically, an upregulation of the expression of extrasynaptic NMDARs was observed, which is supposed to trigger cell death signaling [48, 75-77]. Thus, the early disturbances at synaptic and extrasynaptic sites may cause neurodegeneration in the striatum and consequently behavioral deficits [78]. It is therefore of importance to recapitulate early synaptic perturbations in HD mouse models in order to investigate their involvement in HD pathophysiology and to consider possible early therapies for HD.

The first HD mouse model (R6/2) was developed in 1996 [79]. R6/2 mice, which express a truncated $\mathrm{mHTT}$, are still widely used for studying the pathomechanisms of HD $[34,35,79,80]$. R6/2 mice die around the age of 3-4 months. Two other widely used HD mouse models are YAC128 (expression of full-length human mHTT via a yeast artificial chromosome; [11]) and HdhQ150 (targeted knock-in of chimeric mouse/human mHTT exon1 containing $150 \mathrm{CAG}$ repeats; [36]). These mouse models vary in the way they were generated, how they drive the expression of the mutated HTT, whether they express a truncated or the full-length HTT, CAG repeat length and also in strain background. Consequently, HD symptoms and their progression is different in these mouse models.

Laboratory mice are available in different strain backgrounds. Each strain shows distinct genotypic and phenotypic properties. However, differences within the genome of these strains can influence the phenotype (reviewed in [81]). This can be explained by the expression of modifier-genes that influence the impact of specific gene mutations (reviewed in [21]). The genetic background was already shown to influence the severity of other neurodegenerative diseases [82-85]. As outlined above, variability in occurrence and severity of HD symptoms in HD mouse models can be explained by different lengths of CAG repeats or the type of transgenic model used. However, it is very likely, that the genetic background also influences HD pathology in model mice.

YAC128 mice were originally generated and described in the FVB background [11]. This strain background is known for its high susceptibility to excitotoxicity [86] thus interesting for studying neurodegeneration. However, the C57BL/6 background, is the more widely used background in transgenic mouse models. Mouse lines are often kept in the C57BL/6 (or a mixed) background when sub-breeding two mouse lines. Regarding neurotoxicity, striatal neurons of C57BL/6 mice are, in contrast to those in FVB mice, resistant to quinoline-induced neurodegeneration [87]. We thus wondered whether YAC128 mice in the C57BL/6 background were less susceptible to early synaptic perturbation than YAC128 mice in the FVB background.

Only few studies have so far investigated the YAC128 mouse model in C57BL/6 background [13, 30-33, 88]. A detailed comparison of the behavioral phenotype of the YAC128 
line in the C57BL/6 and FVB background was performed by van Raamsdonk and colleagues [13]. At an age of 12 months, YAC128/FVB mice showed a more severe behavioral phenotype than YAC128/BL6 mice. For example, motor performance on the Rotarod was more impaired and hypoactivity in the Open Field more pronounced in YAC128/FVB then in YAC128/BL6 mice [13]. Deficits of performance in motor and learning tasks are already present in younger YAC128/BL6 mice. For example, performance on the Rotarod is already disturbed at an age of 4 months [31]. Similar to Raamsdonk and colleagues, we observed only mild behavioral deficits in the Open Field and Rotarod in 12-month-old YAC128/BL6 mice. Additionally, we identified subtle changes in the gait of YAC128/BL6 mice. HD patients also suffer from gait abnormalities such as spontaneous knee flexion, changed swing parameters and a decreased step frequency, stride length and velocity [89]. Thus, despite their weaker phenotype and later onset of symptoms YAC128/BL6 mice might be useful for studying early signs of motor deficits in HD. This is of interest as some behavioral experiments cannot be performed with YAC128/FVB background. For example, FVB mice suffer from retinal degeneration, which excludes all behavior experiments involving visual stimuli (https://www.jax.org/strain/027432).

Consistent with the subtle changes in behavior in 12-month-old YAC128/BL6 mice, MSN morphology, spine density, functional synapse number, synapse function, synaptic and extrasynaptic NMDAR-mediated currents, and membrane properties were by and large unaltered. Similar analyses revealed significant alterations in these parameters in YAC128/FVB mice already at a much younger age. For example, the amplitude of extrasynaptic NMDAR-mediated currents increases already in 1-month-old YAC128/FVB mice [48]. In addition, spine density is already reduced in 4-month-old YAC128/FVB mice [39]. In contrast, spine density and amplitude of extrasynaptic NMDAR-mediated currents were not affected in 12-month-old YAC128/BL6 mice. The absence of strong alterations in MSN function is consistent with the low number of inclusion bodies in the striatum of 12month-old YAC128/BL6 mice. Interestingly, it has previously been shown that the mHTT levels is comparable in YAC128 mice in different background strains (FVB, C57BL/6 and 129) [13]. However, the amount of nuclear localization of mHTT is considerably lower in YAC128/BL6 mice than in YAC128/FVB or YAC128/129 mice [13]. Thus, the strain-specific differences in severity of neuropathological alterations do not result from differences in the expression of mHTT but rather from differences in the amount of nuclear localization of mHTT.

HdhQ150/BL6 mice display HD-specific neuropathological alterations already at an age of 6 months. The more severe phenotype of these mice compared to that of YAC128/BL6 mice is consistent with the higher number of inclusion bodies. The alterations in MSN function in HdhQ150/BL6 mice show that mHTT-mediated toxicity occurs also in the C57BL/6 background. That suggests that mHTT is also toxic in the C57BL/6 background, if mHTT is translocated into the nucleus [13].

In conclusion, our data highlight the limitations of the YAC128 mouse model in the C57BL/6 background for the investigation of cellular HD pathologies. Thus, it appears to be favorable to use YAC128 mice in the FVB background. In addition, sufficient numbers of backcrossings are essential when crossing YAC128/FVB mice with transgenic or knockout mice in C57BL/6 background. YAC128 mice in the C57BL/6 background might be useful when investigating HD pathology with behavioral tests that require normal vision. However, the mice should in this case be sufficiently old. Finally, the strain-specific differences in mHTT toxicity may in so far be important as a comparison of gene expression in the different mouse strains could help to identify modifier genes.

\section{Materials and Methods}

\subsection{Mouse lines}


All animal experiments as well as breedings were carried out in compliance with the German Welfare Act and approved by the Governmental Supervisory Panel on Animal Experiments of Baden-Wuerttemberg (G-102/16), Karlsruhe and Rhineland Palatinate, Koblenz (G-16-1-069). All procedures followed the "Principles of laboratory animal care" (NIH publication No. 86-23, revised 1985).

Mice had access to food and water ad libitum and were housed in groups of up to four animals in polycarbonate boxes in an inverted $12 \mathrm{~h}$ light/ $12 \mathrm{~h}$ dark cycle. Cages were changed at least once per week

YAC128 [11]; B6.FVBTg(YAC128)53Hay/ChdiJ; Jackson lab strain 027432) and Hdh (CAG)150 mice, named HdhQ150 in this study (B6.129P2-Htttm2Detl/150J; [36]) were used as mouse models for HD. The YAC128 line was further sub-bred with C57BL/6N mice. YAC128 mice carry a yeast artificial chromosome coding for the human HTT with 128 CAG triplet repeats. The HdhQ150 knock-in mouse model expresses the endogenous mouse HTT with 150 CAG repeats. This mouse model was originally created in a mixed 129/Ola and C57BL/6J background [36] and is now used in 75-90\% C57BL/6 background [10].

Mice of both sexes were used for experiments. Non-transgenic littermates were used as controls in all experimental conditions.

\subsection{Electrophysiology}

YAC128/BL6 and HdhQ150/BL6 mice were anesthetized with 3\% isoflurane and transcardially perfused with oxygenated (95\% O2 / 5\% CO2) ice-cold slicing solution containing (in mM) 10 d-glucose, $26 \mathrm{NaHCO} 3,1.25 \mathrm{NaH} 2 \mathrm{PO} 4,3 \mathrm{KCl}, 7 \mathrm{MgCl}$, 212 sucrose, $0.02 \mathrm{CaCl} 2$ (pH 7.2; 306 mOsm). Using a tissue slicer (VT1200S; Leica, Wetzlar, Germany). Brains were quickly removed and placed into ice-cold solution in the holding chamber of a tissue slicer (Leica, Wetzlar, Germany). 250 $\mu \mathrm{m}$ thick coronal acute brain slices were cut in oxygenated ice-cold artificial cerebrospinal fluid (ACSF) containing (in mM): $125 \mathrm{NaCl}$, 25 d-glucose, $25 \mathrm{NaHCO}$, $1.25 \mathrm{NaH} 2 \mathrm{PO} 4,2.5 \mathrm{KCl}, 2 \mathrm{CaCl} 2,1 \mathrm{MgCl}$; pH 7.2; 306 mOsm. Slices were collected in a holding chamber containing oxygenated ACSF at $37^{\circ} \mathrm{C}$ for 15 min and subsequently at room temperature for $45 \mathrm{~min}$ before recordings.

During recording, slices were constantly perfused with oxygenated ACSF. Neurons were visualized with an Olympus BX51WI upright microscope (Olympus, Shinjuku, Japan) fitted with a $4 x$ objective (Plan N, NA 0.1; Olympus, Shinjuku, Japan), a 40x waterimmersion objective (LUMPlan FI/IR, NA 0.8w; Olympus, Shinjuku, Japan) and a CCD camera (XM10, Olympus, Shinjuku, Japan).

For electrophysiological patch-clamp experiments, recording and stimulation electrodes were controlled by a SM7 remote unit and control box (Luigs \& Neumann, Ratingen, Germany). Glass capillaries (Hilgenberg, Malsfeld, Germany) were filled with the following solution for recording of active and passive membrane properties (in $\mathrm{mM}$ ): 130 K-gluconate, 10 HEPES, 10 phosphocreatine-Na, 10 Na-gluconate, 0.3 GTP, 4 MgATP, 4 $\mathrm{NaCl}$ ( $\mathrm{pH} 7.2$, adjusted with $\mathrm{KOH}$ ). For nucleated patches, glass capillaries were filled with the following solution (in $\mathrm{mM}$ ): $105 \mathrm{~K}$-gluconate, $30 \mathrm{KCl}, 10 \mathrm{HEPES}, 10$ phosphocreatine-Na, $0.3 \mathrm{GTP}, 4 \mathrm{MgATP}$ ( $\mathrm{pH} 7.3$, adjusted with $\mathrm{KOH}$ ). For all other whole-cell recordings the following solution was filled in the glass capillaries (in $\mathrm{mM}$ ): $120 \mathrm{Cs}$-gluconate, $10 \mathrm{CsCl}, 8 \mathrm{NaCl}, 10 \mathrm{HEPES}, 10$ phosphocreatine-Na, 0.3 GTP, 2 MgATP, 0.2 EGTA (pH 7.3 , adjusted with $\mathrm{NaOH}$ ) and $0.1-0.5 \%$ biocytin were additionally added.

Electrical signals were acquired at $10 \mathrm{kHz}$ for miniature excitatory postsynaptic currents (mEPSC) recordings and $50 \mathrm{kHz}$ for all other recordings using an EPC10 amplifier (HEKA, Reutlingen, Germany) and Patchmaster software (HEKA, Reutlingen, Germany). Liquid junction potential was not adjusted. mEPSCs were recorded at a holding potential of $-70 \mathrm{mV}$ in ACSF in the presence of $1 \mu \mathrm{M}$ TTX (Biotrend, Cologne, Germany), hydrobro- 
mide (SR95531; Biotrend, Cologne, Germany) and $50 \mu \mathrm{M}$ APV (Biotrend, Cologne, Germany). Test pulses were delivered every 10 sec to monitor series resistance. Active and passive membrane properties were analyzed by applying hyper- and depolarizing current injections.

Synaptic NMDAR- and AMPAR-mediated currents were evoked using a stimulation electrode (chlorinated silver wire inside a borosilicate glass capillary filled with ACSF), which was located at a distance of approximately $100 \mu \mathrm{m}$ from the patched MSNs. AMPAR- and NMDAR-mediated currents were recorded at holding potentials of -70 and $+40 \mathrm{mV}$, respectively, in ACSF in the presence of hydrobromide (SR95531; Biotrend, Cologne, Germany).

Extrasynaptic NMDAR-mediated currents were analyzed as described previously [90]. Briefly, short pulses $(1 \mathrm{~m})$ of $1 \mathrm{mM}$ glutamate were delivered onto nucleated patches of MSNs with a piezo-controlled theta-glass (Hilgenberg, Malsfeld, Germany) filled with a HEPES solution containing (in $\mathrm{mM}$ ) $135 \mathrm{NaCl}, 10 \mathrm{HEPES}, 5.4 \mathrm{KCl}, 1.8 \mathrm{CaCl}$, 5 glucose, $0.01 \mathrm{CNQX}$, and 0.01 glycine ( $\mathrm{pH} 7.2$ ) as done previously [91]. The piezo actor and control software (Physik Instrumente; Karlsruhe, Germany) were triggered with the Patchmaster software.

NMDAR-mediated current kinetics were obtained from double exponential fits of current deactivation or decay using the formula: $I=A 1(e-t / \tau 1)+A 2(e-t / \tau 2)$, where A1 and A2 are the amplitudes of the time constants $\tau 1$ and $\tau 2$, respectively. The weighted deactivation and decay time constant was calculated as: $\mathrm{w} \tau=\tau 1(A 1 /(A 1+A 2))+\tau 2(A 2 /(A 1+A 2))$

\subsection{Spine and Sholl analysis}

Analysis of neuron anatomy was done as previously described [92]. Briefly, after filling MSNs with an biocytin-containing intercellular solution during electrophysiological analyses, brain slices were removed from the recording chamber, fixed with $4 \%$ Histofix (Roth, Germany) and subsequently stained with Streptavidin-Alexa Fluor 594 (dilution of 1:1000; ThermoFisher Scientific Inc., Waltham, MA, USA). Slices were mounted onto slides with ProLong Gold antifade reagent (ThermoFisher Scientific Inc., Waltham, MA, USA).

Proximal and distal dendrites of MSNs in the striatum were imaged with a $63 \mathrm{x}$ objective (Olympus, Shinjuku, Japan) on a confocal microscope (Leica SP5 LSM; Leica, Wetzlar, Germany). Subsequent blind deconvolution was performed with Amira software (ThermoFisher Scientific Inc., Waltham, MA, USA). Whole-cell images for Sholl analysis were taken with a 40x objective (Olympus, Shinjuku, Japan).

Semi-automatic spine counting of proximal and distal dendrites and tracing of dendrites of whole cells was carried out with NeuronStudio (version 0.9.92; Computational Neurobiology and Imaging Center Mount Sinai School of Medicine, New York, NY, USA). Sholl analyses of the reconstructed neurons was performed with Neurolucida (version 3.70.2; MBF Bioscience; Williston, VT, USA) with starting radius and radius increment parameter set to $10 \mu \mathrm{m}$.

\section{4. mHTT immunostaining}

Mice were deeply anesthetized with isoflurane and transcardially perfused with phosphate buffered saline (PBS) and 4\% PFA (Roth, Karlsruhe, Germany). Then the brain was extracted and cut into coronal mouse brain slices (70 $\mu \mathrm{m}$ thick) with a tissue slicer (VT1000; Leica, Wetzlar, Germany). containing the striatum were fixed in 4\% Histofix (Roth, Germany) and stained with an antibody detecting exclusively huntingtin aggregates as inclusions (monoclonal MW8, dilution 1:10 in 3\% BSA, 2\% NGS, 0.2\% Triton-X100, PBS buffer corresponding to $5 \mu \mathrm{g} / \mathrm{ml}$; Developmental Studies Hybridoma Bank, Iowa 
City, IA, USA). Subsequently, after several washing steps with PBS, the secondary antibody Rabbit anti-Mouse IgG (H+L) Alexa Fluor 594 was added (A27027, dilution of 1:1000, ThermoFisher Scientific Inc., Waltham, MA, USA). Nuclei were visualized using a DAPI staining (D1306, dilution of 1:10.000; ThermoFisher Scientific Inc., Waltham, MA, USA). After several washing steps with PBS, slices were mounted on a glass slide with ProLong Gold antifade reagent (life technologies).

Slices were imaged with a 40x objective (for whole-cell images) or a 63x objective (for dendrite close ups) objective (Leica, Wetzlar, Germany) on a confocal microscope (Leica SP5 LSM; Leica, Wetzlar, Germany).

\subsection{Behavior experiments}

Mice were tested in a battery of behavior experiments: On day 1 mice were tested in the Open Field test and underwent a Rotarod training session. On day 2, mice underwent a second Rotarod training. On day 3, mice performed the Rotarod and Catwalk experiment.

\subsubsection{Open field}

Exploratory behavior and activity of mice was investigated with a standard Open Field test. The box measured $42 \times 42 \mathrm{~cm} 2$ and was subdivided into 16 squares and a squared center area (see Figure 6a). Activity was tracked and analyzed with the SYGNIS tracker software (version 4.14; Sygnis Bioscience, Heidelberg, Germany).

All animals were placed in the same corner and tracked for $5 \mathrm{~min}$. Time as well as the distance travelled in each square was listed. Several parameters were calculated: the total travelled distance; the travelled distance in the periphery (light grey in Figure 6a) versus the travelled distance in the center of the field (dark grey in Figure 6a), the time spent near the wall versus the time spent in the center of the box and the visits spent in the different areas.

\subsubsection{Rotarod}

Mice were trained three times a day on the accelerating Rotarod (Ugi Baslie; Varese, Italy) on two consecutive days. The training was performed under the same conditions as the test trials. The Rotarod was set to accelerate starting from four until 40 rounds per minute within $8 \mathrm{~min}$. Latency to fall off the Rotarod was measured. Mice that did not fall off within 8 min were removed from the Rotarod by hand and their latency to fall was set to $8 \mathrm{~min}$. Each mouse was tested three times with a resting time of $20 \mathrm{~min}$ in between the experiments and the mean of the three time points was calculated.

\subsubsection{Catwalk/Gait analysis}

Gait analysis was performed on a Catwalk as described previously [93]. The Catwalk system consisted of an enclosed corridor $(4.5 \mathrm{~cm}$ broad) with a glass walkway. Fluorescent light is internally reflected within the walkway. Scattering of the light when the mouse paws contact the glass floor produces paw prints. Sessions were recorded and analyzed with the CatWalk XT 10.6 software (Noldus, Wageningen, the Netherlands). For each mouse, three runs with a maximal run duration of $10 \mathrm{~s}$ were recorded and runs with rather constant speed were analyzed (speed variance $<100 \%$ ). The mean of the three time points was calculated for the final gait values. Footprints were classified automatically and adjusted manually, if necessary.

Gait parameters were analyzed as done previously [93]. The swing speed describes the speed of a paw during swing. The swing phase time (swing) describes the time one 
paw is in the air during one step. Support from the abdomen is observed when not only the paws are specified on the glass plate but also the abdomen which can relieve wait from the hint paws [94]. The print of the abdomen was classified automatically as the prints of the paws and was manually adjusted, if necessary. It describes the total duration of abdominal contact with the glass plate (given in \%) over the entire run of the animal.

\subsection{Statistical analysis}

Statistical analyses were performed in GraphPad Prism Version 6.07 (GraphPad Software; San Diego, CA, USA). Data was tested for normal distribution with D'AgostinoPearson omnibus normality test. Normally distributed data were analyzed by $\mathrm{t}$-test, notnormally distributed data were analyzed by a Mann-Whitney-test. Bar graphs show the median \pm interquartile range (IQR) for normally and not-normally distributed data. A pvalue $<0.05$ was considered statistically significant ${ }^{*}=p<0.05,{ }^{* *}=p<0.01,{ }^{* * *}=p<$ $0.001)$.

Supplementary Materials: The following are available online at www.mdpi.com/xxx/s1, Figure S1: Functional synapses are not impaired in 3-month-old and 18-month-old YAC128/BL6 mice

Author Contributions: Conceptualization, J.vE. and MK.B.; data acquisition, S.R.; J.K.; MK.B.; formal analysis, MK.B.; J.K.; S.R.; data curation, MK.B.; writing-original draft preparation, M.B.; J.K.; writing-review and editing, J.vE; MK.B.; J.K.; S.R.; visualization, MK.B.; supervision, MK.B.; project administration, MK.B.; J.vE; funding acquisition, J.vE. All authors have read and agreed to the published version of the manuscript.

Funding: Please add: This research was funded by the Fritz Thyssen foundation (Az 10.15.1.017MN; approval date: 6 July 2017).

Institutional Review Board Statement: The study was conducted according to the guidelines of the Declaration of Helsinki, and approved by the state investigation office of Rhineland Palatinate (G16-1-069; 22 September 2016).

Data Availability Statement: In this section, please provide details regarding where data supporting reported results can be found, including links to publicly archived datasets analyzed or generated during the study. Please refer to suggested Data Availability Statements in section "MDPI Research Data Policies" at https://www.mdpi.com/ethics. You might choose to exclude this statement if the study did not report any data.

Acknowledgments: We thank Barbara Biesalski for technical assistance in preparing brain slices. We also thank the Interdisciplinary Neurobehavioral Core, University of Heidelberg, for training and technical help in behavioral experiments. The MW8 antibody developed by Patterson, P.H.; California Institute of Technology, was obtained from the Developmental Studies Hybridoma Bank, created by the NICHD of the NIH and maintained at The University of Iowa, Department of Biology, Iowa City, IA 52242. We also thank the Microscopy \& Histology Core Facility of the Institute of Molecular Biology gGmbH (IMB) Mainz for technical help with microscopy.

Conflicts of Interest: The authors declare no conflict of interest.

\section{References}

1. Vonsattel, J.P., et al., Neuropathological classification of Huntington's disease. J Neuropathol Exp Neurol, 1985. 44(6): p. 55977.10.1097/00005072-198511000-00003

2. Blumenstock, S. and I. Dudanova, Cortical and Striatal Circuits in Huntington's Disease. Front Neurosci, 2020. 14: p. 82.10.3389/fnins.2020.00082

3. Rub, U., et al., Huntington's disease (HD): the neuropathology of a multisystem neurodegenerative disorder of the human brain. Brain Pathol, 2016. 26(6): p. 726-740.10.1111/bpa.12426

4. Duyao, M., et al., Trinucleotide repeat length instability and age of onset in Huntington's disease. Nat Genet, 1993. 4(4): p. 38792.10.1038/ng0893-387 
5. Zuccato, C., M. Valenza, and E. Cattaneo, Molecular mechanisms and potential therapeutical targets in Huntington's disease. Physiol Rev, 2010. 90(3): p. 905-81.10.1152/physrev.00041.2009

6. $\quad$ Southwell, A.L., et al., A fully humanized transgenic mouse model of Huntington disease. Hum Mol Genet, 2013. 22(1): p. 1834.10.1093/hmg/dds397

7. Caron, N.S., E.R. Dorsey, and M.R. Hayden, Therapeutic approaches to Huntington disease: from the bench to the clinic. Nat Rev Drug Discov, 2018. 17(10): p. 729-750.10.1038/nrd.2018.133

8. Schippling, S., et al., Abnormal motor cortex excitability in preclinical and very early Huntington's disease. Biol Psychiatry, 2009. 65(11): p. 959-65.10.1016/j.biopsych.2008.12.026

9. Cummings, D.M., et al., Aberrant cortical synaptic plasticity and dopaminergic dysfunction in a mouse model of Huntington's disease. Hum Mol Genet, 2006. 15(19): p. 2856-68.10.1093/hmg/ddl224

10. Heng, M.Y., et al., Longitudinal evaluation of the Hdh(CAG)150 knock-in murine model of Huntington's disease. J Neurosci, 2007. 27(34): p. 8989-98.10.1523/JNEUROSCI.1830-07.2007

11. Slow, E.J., et al., Selective striatal neuronal loss in a YAC128 mouse model of Huntington disease. Hum Mol Genet, 2003. 12(13): p. 1555-67.10.1093/hmg/ddg169

12. Marco, S., et al., Suppressing aberrant GluN3A expression rescues synaptic and behavioral impairments in Huntington's disease models. Nat Med, 2013. 19(8): p. 1030-8.10.1038/nm.3246

13. Van Raamsdonk, J.M., et al., Phenotypic abnormalities in the YAC128 mouse model of Huntington disease are penetrant on multiple genetic backgrounds and modulated by strain. Neurobiol Dis, 2007. 26(1): p. 189-200.10.1016/j.nbd.2006.12.010

14. Van Raamsdonk, J.M., et al., Cognitive dysfunction precedes neuropathology and motor abnormalities in the YAC128 mouse model of Huntington's disease. J Neurosci, 2005. 25(16): p. 4169-80.10.1523/JNEUROSCI.0590-05.2005

15. Garcia-Miralles, M., et al., Laquinimod rescues striatal, cortical and white matter pathology and results in modest behavioural improvements in the YAC128 model of Huntington disease. Sci Rep, 2016. 6: p. 31652.10.1038/srep31652

16. Halliday, G.M., et al., Regional specificity of brain atrophy in Huntington's disease. Exp Neurol, 1998. 154(2): p. 66372.10.1006/exnr.1998.6919

17. Cummings, D.M., et al., Alterations in cortical excitation and inhibition in genetic mouse models of Huntington's disease. J Neurosci, 2009. 29(33): p. 10371-86.10.1523/JNEUROSCI.1592-09.2009

18. Crook, Z.R. and D. Housman, Huntington's disease: can mice lead the way to treatment? Neuron, 2011. 69(3): p. 42335.10.1016/j.neuron.2010.12.035

19. Neuner, S.M., et al., Harnessing Genetic Complexity to Enhance Translatability of Alzheimer's Disease Mouse Models: A Path toward Precision Medicine. Neuron, 2019. 101(3): p. 399-411 e5.10.1016/j.neuron.2018.11.040

20. Smith, J.C., A Review of Strain and Sex Differences in Response to Pain and Analgesia in Mice. Comp Med, 2019. 69(6): p. 490500.10.30802/AALAS-CM-19-000066

21. Gusella, J.F., M.E. MacDonald, and J.M. Lee, Genetic modifiers of Huntington's disease. Mov Disord, 2014. 29(11): p. 135965.10.1002/mds. 26001

22. Van Raamsdonk, J.M., et al., Selective degeneration and nuclear localization of mutant huntingtin in the YAC128 mouse model of Huntington disease. Hum Mol Genet, 2005. 14(24): p. 3823-35.10.1093/hmg/ddi407

23. Hamilton, J., T. Brustovetsky, and N. Brustovetsky, Oxidative metabolism and $\mathrm{Ca}(2+)$ handling in striatal mitochondria from YAC128 mice, a model of Huntington's disease. Neurochem Int, 2017. 109: p. 24-33.10.1016/j.neuint.2017.01.001

24. Garcia-Miralles, M., et al., Early pridopidine treatment improves behavioral and transcriptional deficits in YAC128 Huntington disease mice. JCI Insight, 2017. 2(23).10.1172/jci.insight.95665

25. Garcia-Miralles, M., et al., Laquinimod Treatment Improves Myelination Deficits at the Transcriptional and Ultrastructural Levels in the YAC128 Mouse Model of Huntington Disease. Mol Neurobiol, 2019. 56(6): p. 4464-4478.10.1007/s12035-018-1393-1 
26. Petrella, L.I., et al., A whole brain longitudinal study in the YAC128 mouse model of Huntington's disease shows distinct trajectories of neurochemical, structural connectivity and volumetric changes. Hum Mol Genet, 2018. 27(12): p. 21252137.10.1093/hmg/ddy119

27. Silva, A.C., et al., Characterization of subventricular zone-derived progenitor cells from mild and late symptomatic YAC128 mouse model of Huntington's disease. Biochim Biophys Acta Mol Basis Dis, 2018. 1864(1): p. 34-44.10.1016/j.bbadis.2017.09.009

28. Schmidt, M.E., et al., Altering cortical input unmasks synaptic phenotypes in the YAC128 cortico-striatal co-culture model of Huntington disease. BMC Biol, 2018. 16(1): p. 58.10.1186/s12915-018-0526-3

29. Schmidt, M.E., et al., DAPK1 Promotes Extrasynaptic GluN2B Phosphorylation and Striatal Spine Instability in the YAC128 Mouse Model of Huntington Disease. Front Cell Neurosci, 2020. 14: p. 590569.10.3389/fncel.2020.590569

30. Bayram-Weston, Z., et al., Light and electron microscopic characterization of the evolution of cellular pathology in YAC128 Huntington's disease transgenic mice. Brain Res Bull, 2012. 88(2-3): p. 137-47.10.1016/j.brainresbull.2011.05.005

31. Brooks, S., et al., Longitudinal analysis of the behavioural phenotype in YAC128 (C57BL/6J) Huntington's disease transgenic mice. Brain Res Bull, 2012. 88(2-3): p. 113-20.10.1016/j.brainresbull.2010.05.005

32. Brooks, S.P., et al., Selective cognitive impairment in the YAC128 Huntington's disease mouse. Brain Res Bull, 2012. 88(2-3): p. 121-9.10.1016/j.brainresbull.2011.05.010

33. Brooks, S.P., L. Jones, and S.B. Dunnett, Longitudinal analyses of operant performance on the serial implicit learning task (SILT) in the YAC128 Huntington's disease mouse line. Brain Res Bull, 2012. 88(2-3): p. 130-6.10.1016/j.brainresbull.2011.06.008

34. Menalled, L., et al., Systematic behavioral evaluation of Huntington's disease transgenic and knock-in mouse models. Neurobiol Dis, 2009. 35(3): p. 319-36.10.1016/j.nbd.2009.05.007

35. Cummings, D.M., C. Cepeda, and M.S. Levine, Alterations in striatal synaptic transmission are consistent across genetic mouse models of Huntington's disease. ASN Neuro, 2010. 2(3): p. e00036.10.1042/AN20100007

36. Lin, C.H., et al., Neurological abnormalities in a knock-in mouse model of Huntington's disease. Hum Mol Genet, 2001. 10(2): p. 137-44.10.1093/hmg/10.2.137

37. Ferrante, R.J., N.W. Kowall, and E.P. Richardson, Jr., Proliferative and degenerative changes in striatal spiny neurons in Huntington's disease: a combined study using the section-Golgi method and calbindin D28k immunocytochemistry. J Neurosci, 1991. 11(12): p. 3877-87, https://www.ncbi.nlm.nih.gov/pubmed/1836019

38. Wu, J., et al., Enhanced Store-Operated Calcium Entry Leads to Striatal Synaptic Loss in a Huntington's Disease Mouse Model. J Neurosci, 2016. 36(1): p. 125-41.10.1523/JNEUROSCI.1038-15.2016

39. Madison, J.L., et al., Disease-toxicant interactions in manganese exposed Huntington disease mice: early changes in striatal neuron morphology and dopamine metabolism. PLoS One, 2012. 7(2): p. e31024.10.1371/journal.pone.0031024

40. Paulsen, J.S., et al., Detection of Huntington's disease decades before diagnosis: the Predict-HD study. J Neurol Neurosurg Psychiatry, 2008. 79(8): p. 874-80.10.1136/jnnp.2007.128728

41. Orth, M., et al., Observing Huntington's Disease: the European Huntington's Disease Network's REGISTRY. PLoS Curr, 2010. 2: p. RRN1184.10.1371/currents.RRN1184

42. Andre, V.M., et al., Differential electrophysiological changes in striatal output neurons in Huntington's disease. J Neurosci, 2011. 31(4): p. 1170-82.10.1523/JNEUROSCI.3539-10.2011

43. Andre, V.M., Y.E. Fisher, and M.S. Levine, Altered Balance of Activity in the Striatal Direct and Indirect Pathways in Mouse Models of Huntington's Disease. Front Syst Neurosci, 2011. 5: p. 46.10.3389/fnsys.2011.00046

44. Kolodziejczyk, K. and L.A. Raymond, Differential changes in thalamic and cortical excitatory synapses onto striatal spiny projection neurons in a Huntington disease mouse model. Neurobiol Dis, 2016. 86: p. 62-74.10.1016/j.nbd.2015.11.020

45. Joshi, P.R., et al., Age-dependent alterations of corticostriatal activity in the YAC128 mouse model of Huntington disease. J Neurosci, 2009. 29(8): p. 2414-27.10.1523/JNEUROSCI.5687-08.2009 
46. Cepeda, C., et al., NMDA receptor function in mouse models of Huntington disease. J Neurosci Res, 2001. 66(4): p. 52539.10.1002/jnr.1244

47. Graham, R.K., et al., Differential susceptibility to excitotoxic stress in YAC128 mouse models of Huntington disease between initiation and progression of disease. J Neurosci, 2009. 29(7): p. 2193-204.10.1523/JNEUROSCI.5473-08.2009

48. Milnerwood, A.J., et al., Early increase in extrasynaptic NMDA receptor signaling and expression contributes to phenotype onset in Huntington's disease mice. Neuron, 2010. 65(2): p. 178-90.10.1016/j.neuron.2010.01.008

49. Fan, M.M. and L.A. Raymond, N-methyl-D-aspartate (NMDA) receptor function and excitotoxicity in Huntington's disease. Prog Neurobiol, 2007. 81(5-6): p. 272-93.10.1016/j.pneurobio.2006.11.003

50. Raymond, L.A., et al., Pathophysiology of Huntington's disease: time-dependent alterations in synaptic and receptor function. Neuroscience, 2011. 198: p. 252-73.10.1016/j.neuroscience.2011.08.052

51. Landwehrmeyer, G.B., et al., NMDA receptor subunit mRNA expression by projection neurons and interneurons in rat striatum. $\mathrm{J}$ Neurosci, 1995. 15(7 Pt 2): p. 5297-307, https://www.ncbi.nlm.nih.gov/pubmed/7623152

52. Stroebel, D., M. Casado, and P. Paoletti, Triheteromeric NMDA receptors: from structure to synaptic physiology. Curr Opin Physiol, 2018. 2: p. 1-12.10.1016/j.cophys.2017.12.004

53. Vicini, S., et al., Functional and pharmacological differences between recombinant N-methyl-D-aspartate receptors. J Neurophysiol, 1998. 79(2): p. 555-66.10.1152/jn.1998.79.2.555

54. Hardingham, G.E. and H. Bading, Coupling of extrasynaptic NMDA receptors to a CREB shut-off pathway is developmentally regulated. Biochim Biophys Acta, 2002. 1600(1-2): p. 148-53.10.1016/s1570-9639(02)00455-7

55. Bading, H., Therapeutic targeting of the pathological triad of extrasynaptic NMDA receptor signaling in neurodegenerations. J Exp Med, 2017. 214(3): p. 569-578.10.1084/jem.20161673

56. Woodman, B., et al., The Hdh(Q150/Q150) knock-in mouse model of HD and the R6/2 exon 1 model develop comparable and widespread molecular phenotypes. Brain Res Bull, 2007. 72(2-3): p. 83-97.10.1016/j.brainresbull.2006.11.004

57. Cepeda, C., et al., Transient and progressive electrophysiological alterations in the corticostriatal pathway in a mouse model of Huntington's disease. J Neurosci, 2003. 23(3): p. 961-9, https://www.ncbi.nlm.nih.gov/pubmed/12574425

58. Indersmitten, T., et al., Altered excitatory and inhibitory inputs to striatal medium-sized spiny neurons and cortical pyramidal neurons in the Q175 mouse model of Huntington's disease. J Neurophysiol, 2015. 113(7): p. 2953-66.10.1152/jn.01056.2014

59. Davies, S.W. and E. Scherzinger, Nuclear inclusions in Huntington's disease. Trends Cell Biol, 1997. 7(11): p. 422.10.1016/S09628924(97)88136-6

60. Usdin, M.T., et al., Impaired synaptic plasticity in mice carrying the Huntington's disease mutation. Hum Mol Genet, 1999. 8(5): p. 839-46.10.1093/hmg/8.5.839

61. Lawrence, A.D., et al., Visual object and visuospatial cognition in Huntington's disease: implications for information processing in corticostriatal circuits. Brain, 2000. 123 ( Pt 7): p. 1349-64.10.1093/brain/123.7.1349

62. Berrios, G.E., et al., Psychiatric symptoms and CAG repeats in neurologically asymptomatic Huntington's disease gene carriers. Psychiatry Res, 2001. 102(3): p. 217-25.10.1016/s0165-1781(01)00257-8

63. Mazarakis, N.K., et al., Deficits in experience-dependent cortical plasticity and sensory-discrimination learning in presymptomatic Huntington's disease mice. J Neurosci, 2005. 25(12): p. 3059-66.10.1523/JNEUROSCI.4320-04.2005

64. Milnerwood, A.J., et al., Early development of aberrant synaptic plasticity in a mouse model of Huntington's disease. Hum Mol Genet, 2006. 15(10): p. 1690-703.10.1093/hmg/dd1092

65. Paulsen, J.S., et al., Brain structure in preclinical Huntington's disease. Biol Psychiatry, 2006. 59(1): p. 5763.10.1016/j.biopsych.2005.06.003

66. Duff, K., et al., Psychiatric symptoms in Huntington's disease before diagnosis: the predict-HD study. Biol Psychiatry, 2007. 62(12): p. 1341-6.10.1016/j.biopsych.2006.11.034 
67. Solomon, A.C., et al., Verbal episodic memory declines prior to diagnosis in Huntington's disease. Neuropsychologia, 2007. 45(8): p. 1767-76.10.1016/j.neuropsychologia.2006.12.015

68. Levine, M.S., et al., Enhanced sensitivity to N-methyl-D-aspartate receptor activation in transgenic and knockin mouse models of Huntington's disease. J Neurosci Res, 1999. 58(4): p. 515-32, https://www.ncbi.nlm.nih.gov/pubmed/10533044

69. Hansson, O., et al., Resistance to NMDA toxicity correlates with appearance of nuclear inclusions, behavioural deficits and changes in calcium homeostasis in mice transgenic for exon 1 of the huntington gene. Eur J Neurosci, 2001. 14(9): p. 1492-504.10.1046/j.0953816x.2001.01767.x

70. Laforet, G.A., et al., Changes in cortical and striatal neurons predict behavioral and electrophysiological abnormalities in a transgenic murine model of Huntington's disease. J Neurosci, 2001. 21(23): p. 9112-23, https://www.ncbi.nlm.nih.gov/pubmed/11717344

71. Zeron, M.M., et al., Increased sensitivity to N-methyl-D-aspartate receptor-mediated excitotoxicity in a mouse model of Huntington's disease. Neuron, 2002. 33(6): p. 849-60.10.1016/s0896-6273(02)00615-3

72. Starling, A.J., et al., Alterations in N-methyl-D-aspartate receptor sensitivity and magnesium blockade occur early in development in the R6/2 mouse model of Huntington's disease. J Neurosci Res, 2005. 82(3): p. 377-86.10.1002/jnr.20651

73. Shehadeh, J., et al., Striatal neuronal apoptosis is preferentially enhanced by NMDA receptor activation in YAC transgenic mouse model of Huntington disease. Neurobiol Dis, 2006. 21(2): p. 392-403.10.1016/j.nbd.2005.08.001

74. Yohrling, I.G., et al., Inhibition of tryptophan hydroxylase activity and decreased 5-HT1A receptor binding in a mouse model of Huntington's disease. J Neurochem, 2002. 82(6): p. 1416-23.10.1046/j.1471-4159.2002.01084.x

75. Milnerwood, A.J., et al., Mitigation of augmented extrasynaptic NMDAR signaling and apoptosis in cortico-striatal co-cultures from Huntington's disease mice. Neurobiol Dis, 2012. 48(1): p. 40-51.10.1016/j.nbd.2012.05.013

76. Levine, M.S., C. Cepeda, and V.M. Andre, Location, location, location: contrasting roles of synaptic and extrasynaptic NMDA receptors in Huntington's disease. Neuron, 2010. 65(2): p. 145-7.10.1016/j.neuron.2010.01.010

77. Dau, A., et al., Chronic blockade of extrasynaptic NMDA receptors ameliorates synaptic dysfunction and pro-death signaling in Huntington disease transgenic mice. Neurobiol Dis, 2014. 62: p. 533-42.10.1016/j.nbd.2013.11.013

78. Milnerwood, A.J. and L.A. Raymond, Corticostriatal synaptic function in mouse models of Huntington's disease: early effects of huntingtin repeat length and protein load. J Physiol, 2007. 585(Pt 3): p. 817-31.10.1113/jphysiol.2007.142448

79. Mangiarini, L., et al., Exon 1 of the HD gene with an expanded CAG repeat is sufficient to cause a progressive neurological phenotype in transgenic mice. Cell, 1996. 87(3): p. 493-506.10.1016/s0092-8674(00)81369-0

80. Bates, G.P. and E. Hockly, Experimental therapeutics in Huntington's disease: are models useful for therapeutic trials? Curr Opin Neurol, 2003. 16(4): p. 465-70.10.1097/01.wco.0000084223.82329.bb

81. Taft, R.A., M. Davisson, and M.V. Wiles, Know thy mouse. Trends Genet, 2006. 22(12): p. 649-53.10.1016/j.tig.2006.09.010

82. Heiman-Patterson, T.D., et al., Background and gender effects on survival in the TgN(SOD1-G93A)1Gur mouse model of ALS. J Neurol Sci, 2005. 236(1-2): p. 1-7.10.1016/j.jns.2005.02.006

83. Monani, U.R., D.D. Coovert, and A.H. Burghes, Animal models of spinal muscular atrophy. Hum Mol Genet, 2000. 9(16): p. 2451-7.10.1093/hmg/9.16.2451

84. Heiman-Patterson, T.D., et al., Effect of genetic background on phenotype variability in transgenic mouse models of amyotrophic lateral sclerosis: a window of opportunity in the search for genetic modifiers. Amyotroph Lateral Scler, 2011. 12(2): p. 7986.10.3109/17482968.2010.550626

85. Coley, W.D., et al., Effect of genetic background on the dystrophic phenotype in mdx mice. Hum Mol Genet, 2016. 25(1): p. 13045.10.1093/hmg/ddv460

86. Schauwecker, P.E. and O. Steward, Genetic determinants of susceptibility to excitotoxic cell death: implications for gene targeting approaches. Proc Natl Acad Sci U S A, 1997. 94(8): p. 4103-8.10.1073/pnas.94.8.4103

87. McLin, J.P., L.M. Thompson, and O. Steward, Differential susceptibility to striatal neurodegeneration induced by quinolinic acid and kainate in inbred, outbred and hybrid mouse strains. Eur J Neurosci, 2006. 24(11): p. 3134-40.10.1111/j.1460-9568.2006.05198.x 
88. Bayram-Weston, Z., et al., Light and electron microscopic characterization of the evolution of cellular pathology in the Hdh(CAG)150 Huntington's disease knock-in mouse. Brain Res Bull, 2012. 88(2-3): p. 189-98.10.1016/j.brainresbull.2011.03.014

89. Koller, W.C. and J. Trimble, The gait abnormality of Huntington's disease. Neurology, 1985. 35(10): p. 14504.10.1212/wnl.35.10.1450

90. Muller, M.K., et al., NMDA receptors mediate synaptic depression, but not spine loss in the dentate gyrus of adult amyloid Beta (Abeta) overexpressing mice. Acta Neuropathol Commun, 2018. 6(1): p. 110.10.1186/s40478-018-0611-4

91. Chen, X., et al., CKAMP44 modulates integration of visual inputs in the lateral geniculate nucleus. Nat Commun, 2018. 9(1): p. 261.10.1038/s41467-017-02415-1

92. Back, M.K., et al., Amyloid Beta-Mediated Changes in Synaptic Function and Spine Number of Neocortical Neurons Depend on NMDA Receptors. Int J Mol Sci, 2021. 22(12).10.3390/ijms22126298

93. Vandeputte, C., et al., Automated quantitative gait analysis in animal models of movement disorders. BMC Neurosci, 2010. 11: p. 92.10.1186/1471-2202-11-92

94. Hamers, F.P., et al., Automated quantitative gait analysis during overground locomotion in the rat: its application to spinal cord contusion and transection injuries. J Neurotrauma, 2001. 18(2): p. 187-201.10.1089/08977150150502613 